\title{
NOTE ON RELATIVE $p$-BASES OF PURELY INSEPARABLE EXTENSIONS
}

J. N. MORDESON AND B. VINOGRADE

Throughout this note $L / K$ denotes a purely inseparable field extension of characteristic $p$ and nonzero exponent. In [5, p. 745], Rygg proves that when $L / K$ has bounded exponent, then a subset $M$ of $L$ is a relative $p$-base of $L / K$ if and only if $M$ is a minimal generating set of $L / K$. The purpose of this note is to answer the following question: If every relative $p$-base of $L / K$ is a minimal generating set, then must $L / K$ be of bounded exponent? The answer is known to be yes when $K$ and $L^{p^{i}}$ are linearly disjoint, $i=1,2, \cdots$, see [1]. We give two examples for which the answer is no: One in which the maximal perfect subfield of $L$ is contained in $K$, and the other in which it is not.

The following lemmas are needed for our examples. An intermediate field $L^{\prime}$ of $L / K$ is called proper if $K \subseteq L^{\prime} \subset L$.

Lemma 1. Every relative p-base of $L / K([2, p .180])$ is a minimal generating set of $L / K$ if and only if there does not exist a proper intermediate field $L^{\prime}$ of $L / K$ such that $L=L^{\prime}\left(L^{p}\right)$.

Proof. If $L=L^{\prime}\left(L^{p}\right)$, where $L^{\prime}$ is a proper intermediate field of $L / K$, then $L^{\prime}$ contains a relative $p$-base $M$ of $L / K$. Thus $L \supset L^{\prime}$ $\supseteq K(M)$. Conversely, if there exists a relative $p$-base $M$ of $L / K$ such that $L \supset K(M)$, then $L=L^{\prime}\left(L^{p}\right)$, where $L^{\prime}=K(M)$. Q.E.D.

Lemma 2. Suppose $L=K\left(m_{1}, m_{2}, \cdots\right)$, where $m_{i} \in K\left(m_{i+1}\right)$, $i=1,2, \cdots$. Then $K, K\left(m_{i}^{p^{j_{i}}}\right), L$ are the intermediate fields of $L / K$, $0 \leqq j_{i}<e_{i}\left(e_{i}\right.$ the exponent of $m_{i}$ over $\left.K\left(m_{i-1}\right)\right), i=1,2, \cdots$, where $K\left(m_{0}\right)$ means $K$.

Proof. Let $e_{i}^{\prime}$ denote the exponent of $m_{i}$ over $K, i=1,2, \cdots$ By [2, p. 196, Exercise 5], the intermediate fields of $K\left(m_{s}\right) / K$ are $K\left(m_{s}^{p_{s}}\right), 0 \leqq j_{s} \leqq e_{s}^{\prime}$. If $0<t<s$, then $K\left(m_{t}\right) \subset K\left(m_{s}\right)$, whence $K\left(m_{t}\right)$ $=K\left(m_{s}^{p^{e^{\prime}}{ }^{-e^{\prime}} t_{t}}\right)$. Thus the intermediate fields of $K\left(m_{s}\right) / K$ are $K, K\left(m_{i}^{p_{i}}\right)$, $0 \leqq j_{i}<e_{i}, i=1, \cdots, s$. Let $K^{\prime}$ be any intermediate field of $L / K$. If $\left[K^{\prime}: K\right]<\infty$, then $K^{\prime} / K$ is finitely generated. Hence $K^{\prime} \subseteq K\left(m_{s}\right)$ for some $m_{s}$ since $L=\bigcup_{i=1}^{\infty} K\left(m_{i}\right)$. Thus $K^{\prime}=K\left(m_{i}^{p_{i}}\right)$ for some $m_{i}$ by the preceding argument. If $\left[K^{\prime}: K\right]=\infty$, then $K^{\prime}$ is the union over $c$ of $K(c)$ for all $c \in K^{\prime}$. Now $K(c)=K\left(m_{i_{c}}^{p^{i_{i}}}\right) \supset K\left(m_{i_{c}-1}\right)$ for some $m_{i_{c}}$

Received by the editors January 10, 1969. 
and for all $c \in K^{\prime}-K$ by the previous argument. Since $\left[K^{\prime}: K\right]=\infty$, $i_{c}$ is an unbounded function of $c$. Thus $K^{\prime}=L$. Q.E.D.

EXAMPLE 1. $L / K$ is of unbounded exponent, the maximal perfect subfield of $L$ is not contained in $K$, and every relative $p$-base of $L / K$ is a minimal generating set of $L / K$ : Let $P$ be a perfect field and $z, y, x_{1}, x_{2}, \cdots$ independent indeterminates over $P$. Let $K$ $=P\left(z, y, x_{1}, x_{2}, \cdots\right)$ and $L=K\left(m_{1}, m_{2}, \cdots\right)$, where $m_{i}=z^{p-i-1} x_{i}^{p-1}$ $+y^{p-1}, i=1,2, \cdots$. Clearly, $L / K$ is of unbounded exponent. $P\left(z^{p-1}, z^{p-2}, \cdots\right)$ is the maximal perfect subfield of $L$ and is not in $K$. By Lemma 1 , every relative $p$-base of $L / K$ is a minimal generating set of $L / K$ if we show that $L \neq L^{\prime}\left(L^{p}\right)$ for any proper intermediate field $L^{\prime}$ of $L / K$. We postpone this proof.

EXAmple $2 . L / K$ is of unbounded exponent, the maximal perfect subfield of $L$ is contained in $K$, and every relative $p$-base of $L / K$ is a minimal generating set of $L / K$ : Let $P$ be a perfect field and $y, x_{1}$, $x_{2}, \cdots$ independent indeterminates over $P$. Let $K=P\left(y, x_{1}, x_{2}, \cdots\right)$ and $L=K\left(m_{1}, m_{2}, \cdots\right)$, where $m_{1}=x_{1}^{p^{-2}}$ and $m_{i+1}=\left(m_{i}^{p} y+x_{i+1}\right)^{p^{-2}}$, $i=1,2, \cdots$. Clearly, $L / K$ is of unbounded exponent. It follows that $L=P\left(y, m_{1}, m_{2}, \cdots\right)$ and that $\left\{y, m_{1}, m_{2}, \cdots\right\}$ is an algebraically independent set over $P$. That is, $L / P$ is a pure transcendental extension. Thus $P, P \subseteq K$, is the maximal perfect subfield of $L$ by Corollary 2 of $[3$, p. 388]. By Lemma 1 , it remains to be shown that $L \neq L^{\prime}\left(L^{p}\right)$ for any proper intermediate field $L^{\prime}$ of $L / K$.

We prove simultaneously for Examples 1 and 2 that such a field $L^{\prime}$ cannot exist. In both examples, it follows that $K\left(L^{p}\right)=K\left(m_{1}^{p}, m_{2}^{p}, \cdots\right)$, $m_{i}^{p} \in K\left(m_{i+1}^{p}\right)$ and $m_{i+1}^{p}$ has exponent 1 over $K\left(m_{i}^{p}\right)$ for $i=1,2, \cdots$. Hence, by Lemma 2, the intermediate fields of $K\left(L^{p}\right) / K$ appear in a chain. Now suppose there exists a proper intermediate field $L^{\prime}$ of $L / K$ such that $L=L^{\prime}\left(L^{p}\right)$. Since $L^{\prime} \nsupseteq K\left(L^{p}\right), L^{\prime} \cap K\left(L^{p}\right)=K\left(m_{s}^{p}\right)$ for some integer $s \geqq 0$. We show $L^{\prime}$ and $K\left(L^{p}\right)$ are linearly disjoint over $K\left(m_{s}^{p}\right)$ by showing that for every proper intermediate field $K^{\prime}$ of $K\left(L^{p}\right) / K\left(m_{s}^{p}\right), L^{\prime}$ and $K^{\prime}$ are linearly disjoint over $K\left(m_{s}^{p}\right)$. By Lemma 2, $K^{\prime}=K\left(m_{l}^{p}\right)$ for $t \geqq s$. Now $m_{t}^{p}$ has exponent $t-s$ over $K\left(m_{s}^{p}\right) \subseteq L^{\prime}$. If $\left(\left(m_{l}^{p}\right)^{p t-s}\right)^{p^{-1}} \in L^{\prime}$, then we contradict $L^{\prime} \cap K\left(L^{p}\right)=K\left(m_{s}^{p}\right)$. Hence the irreducible polynomial of $m_{t}^{p}$ over $K\left(m_{s}^{p}\right)$ remains irreducible over $L^{\prime}$. Thus $L^{\prime}$ and $K\left(m_{l}^{p}\right)$ are linearly disjoint over $K\left(m_{s}^{p}\right)$, whence $L^{\prime}$ and $K\left(L^{p}\right)$ are linearly disjoint.

Since $L=L^{\prime}\left(L^{p}\right), m_{s+1} \in L^{\prime}\left(L^{p}\right)$. Hence $m_{s+1}=\sum_{j_{j-0}{ }^{t-1}} c_{j}^{\prime}\left(m_{\imath}^{p}\right)^{j}$ for some integer $t$, where $c_{j}^{\prime} \in L^{\prime}$. Now $t \geqq s+2$ since $m_{s+1}$ has exponent 2 over $K\left(m_{s}^{p}\right)$ and $L^{\prime}$ has exponent 1 over $K\left(m_{s}^{p}\right)$. Thus

$$
m_{s+1}^{p}=\sum_{j} c_{j}^{\prime p}\left(m_{t}^{p}\right)^{j p}
$$


By the division algorithm, $j p=p^{t-\triangleleft} q_{j}+r_{j}, 0 \leqq r_{j}<p^{t-s}$. Hence

$$
m_{s+1}^{p}=\sum_{j}\left(c_{j}^{p p}\left(m_{t}^{p}\right)^{p^{t-c_{q}} q_{j}}\right)\left(m_{t}^{p}\right)^{r_{j}}
$$

and

$$
c_{j}^{\prime p}\left(m_{t}^{p}\right)^{p^{t-q_{j}} q_{j}}=c_{j}^{p} \in L^{\prime} \cap L^{p} .
$$

Writing $m_{s+1}^{p}$ in terms of $m_{t}^{p}$, we get for Example 1:

$$
m_{s+1}^{p}=\left(m_{t}^{p}\right)^{p^{t-\sigma-1}} k_{0}^{p} x_{s+1}-k_{1}
$$

where

$$
k_{0}^{p}=x_{t}^{-p^{t-\sigma-1}}
$$

and

$$
k_{1}=y^{p^{t-\sigma-1}} x_{t}^{-p^{t-\sigma-1}} x_{8+1}-y .
$$

By $\left(^{*}\right)$,

$$
\left(m_{t}^{p}\right)^{p^{t-o-1}} x_{s+1}=k_{0}^{-p} k_{1}+k_{0}^{-p} \sum_{j} c_{j}^{p}\left(m_{t}^{p}\right)^{r_{j}} .
$$

Hence, by the linear disjointness of $L^{\prime}$ and $K\left(L^{p}\right)$ over $K\left(m_{s}^{p}\right)$ and since $\left\{\left(m_{l}^{p}\right)^{j} \mid j=0, \cdots, p^{t-s}-1\right\}$ is linearly independent over $K\left(m_{s}^{p}\right)$, $x_{s+1} \in L^{\prime} \cap L^{p}$. Thus $x_{s+1}^{p^{-1}} \in L$, a contradiction.

For Example 2, we get

$$
m_{s+1}^{p}=\left(m_{s+2}^{p}\right)^{p} y^{-1}-x_{s+2} y^{-1}=\cdots=\left(m_{t}^{p}\right)^{p^{t--1}} k_{0}^{p} y^{-1}-k_{1}
$$

for suitable $k_{0}, k_{1} \in K$. By an argument similar to that in Example 1, we obtain $y^{p^{-1}} \in L$, a contradiction.

REMARK. Let $P$ denote the maximal perfect subfield of $L$ and $M$ a relative $p$-base of $L / K$. Consider the properties: (1) $P \subseteq K$, (2) $P \nsubseteq K$, (3) there exists an $M$ such that $L=K(M)$, and (4) for all $M, L=K(M)$. None implies the other except for (4) implies (3).

For instance, Example 1 shows that $(4) \rightarrow(1)$ and Example 2 shows that $(4) \rightarrow(2)$. Example 2 of $[4$, p. 333] shows that $(3) \rightarrow(4)$. Letting $L$ be perfect gives us an example showing that $(2) \rightarrow(3)$. We show $(1) \rightarrow(3)$ by giving an example constructed by $E$. A. Hamann. Let $K=Q\left(x_{1}, x_{2}, \cdots\right)$ and

$$
L=K\left(x_{1}^{p^{-1}},\left(x_{1}+x_{2}^{p}\right)^{p^{-2}},\left(x_{1}+x_{2}^{p}+x_{1}^{p^{2}}\right)^{p^{-3}}, \cdots\right),
$$

where $Q$ is a perfect field and $x_{1}, x_{2}, \ldots$ are independent indeterminates over $Q .\left(L=K\left(L^{p}\right)\right.$ and $L / Q$ is pure transcendental.) The remaining implications are trivial. 


\section{REFERENCES}

1. G. Haddix, J. Mordeson and B. Vinograde, On purely inseparable extensions of unbounded exponent, Canad. J. Math. (to appear).

2. N. Jacobson, Lectures in abstract algebra. Vol. III, Van Nostrand, Princeton, N. J., 1964.

3. S. MacLane, Modular fields, Duke Math. J. 5 (1939), 372-393.

4. J. Mordeson and B. Vinograde, Generators and tensor factors of purely inseparable fields, Math. Z. 107 (1968), 326-334.

5. P. Rygg, On minimal sets of generators of purely inseparable field extensions, Proc. Amer. Math. Soc. 14 (1963), 742-745.

Creighton University AND

Iowa State University 